\title{
Antidoping analysis: a special focus
}

\author{
David A Cowan*,1 (iD \\ ${ }^{1}$ Department of Analytical, Environmental \& Forensic Sciences, King's College London, 150 Stamford Street, London SE1 9NH, UK \\ *Author for correspondence: david.a.cowan@kcl.ac.uk
}

First draft submitted: 13 May 2020; Accepted for publication: 28 May 2020; Published online: 8 July 2020 Keywords: biomarkers in antidoping $\bullet$ gene doping $\bullet$ metabolomics in antidoping $\bullet$ peptide quantification $\bullet$ VAMS
in antidoping

This issue of Bioanalysis has the theme of antidoping analysis and was originally planned to be published to coincide with the run-up to the 2020 Olympic and Paralympic Games in Tokyo, Japan. It was intended to give the interested reader an indication of the latest approaches used by the scientists working in the antidoping field that would likely be used during the Games in the Tokyo World Anti-Doping Agency (WADA) accredited antidoping laboratory. The COVID-19 pandemic has affected all walks of life internationally and sport is no exception; hence the games have been postponed for a year until 2021 by the International Olympic Committee, for the first time ever. The ability, under WADA and International Olympic Committee regulations, to store samples for up to 10 years means that even if some of the approaches described in this issue are not yet ready for use to take action against a cheating athlete, retrospective analysis could enable this to occur at some time in the years ahead.

I have found the articles in this issue always interesting to read and informative, being written by and with the opinions of experts in the field of antidoping science. The conclusions or opinions documented should provide fertile ground for further research. Here is a summary of what you will find.

Aikin and colleagues from WADA provide a well-informed perspective about the current and future use of biomarkers in antidoping testing with a special focus on athlete biological passports pioneered by their organization. They outline what is required and make it clear that discovering new biomarkers and implementing their use is no easy task, but worth the effort in making antidoping testing more effective [1].

Botré and colleagues provide a commentary on the need for a metabolomics approach to deal with some of the more complex analytical challenges in antidoping, and the potential of the approach for use in the field. However, they point out numerous challenges and conclude that "stand-alone screening + confirmation techniques based on metabolomics approaches still seem premature in antidoping," but indicate that explorative metabolomics are of value even now to help identify putative biomarkers [2].

An update on the state of play of peptide quantification in antidoping and how it often links with the requirements of clinical science is provided in an editorial by Moncrieffe. The need for traceable reference standards is stressed and the importance of LC-MS to provide the 'gold-standard' approach contrasting with some of the problems encountered with the use of immuno-procedures. She stresses the benefits of and need for good interlaboratory studies to show the reliability and robustness of the methods [3].

Fedoruk of the United States Anti-Doping Agency (USADA) presents in his editorial how USADA has modified its approach to antidoping testing as a result of the COVID-19 pandemic [4]. He explains how athletes attempt to beat the testers and some of the actions used to prevent this. USADA have piloted a self-testing program, known as the 'virtual drug testing program' where sample collection personnel monitor the process remotely using audio and video from mobile devices. In addition to urine collection, using currently available modern simple phlebotomy devices enables athletes to collect their own blood samples as well. In my opinion, this is an excellent example of how a resourceful organization can react to adversity, making best use of the latest science.

Pizzatti and colleagues connected with the Olympic antidoping laboratory in Rio de Janeiro review the various gene doping strategies and possible target tissues. They conclude that, with the advances in techniques in the field of molecular biology, the possibilities for detecting gene doping increase accordingly [5]. 
As a key researcher in developing one of the approaches to detect gene doping, Baoutina is eminently placed to provide a commentary on the development of a gene doping test and, indeed, that is just what she has done in a form that is understandable to any intelligent scientist without the need to be a trained geneticist. In-line with the view of Pizzatti and colleagues, she starts her article with the question that people may, very understandably, ask why a test hasn't been already implemented. She then clearly explains the issues faced by the scientist in bringing such novel technologies into a robust and legally defensible method, which is useable by WADA-accredited antidoping laboratories [6].

The research by Marchand and colleagues confirms that dried blood collected as spots on cards or by using volumetric absorptive microsampling tips with a $20 \mu \mathrm{l}$ sample capacity can be used for IGF-I quantification with results that correlate well with blood serum concentrations [7].

Meanwhile, the research by Mercolini and colleagues suggests that analyzing dried urine samples, either as spots on cards or again using volumetric absorptive microsampling devices with a $30 \mu \mathrm{l}$ volume, has a number of benefits for antidoping analysis compared with the current method of transporting liquid urine samples. They contrast the benefits of stability of the analytes with the disadvantage of insufficient sensitivity. Nevertheless, they are of the opinion that this is an approach worth pursuing. Clearly, working with dried samples makes transportation simpler and cheaper [8].

Leuenberger and colleagues have shown that an automated method of analysis they used for transcriptomic biomarkers from reticulocytes in dried blood spots was as good as or better than manual sample handling. They applied their methodology to examine changes in the expression levels of a number of factors in samples from elite athletes and volunteers after recombinant erythropoietin injection [9].

Scientists from the China AntiDoping Agency, led by Lu, provide a timely and much needed research paper relating to evidencing the administration of pharmaceutical clenbuterol from inadvertent administration via eating contaminated meat from animals that have been illegally dosed with this lipolytic drug. Their work shows that ethnic differences may influence the enantiomeric elimination of the drug and hence special care is needed in the interpretation of the analytical results if using these in the determination of a case where clenbuterol has been found in the urine of an athlete [10].

Finally, the work of Nicoli and colleagues confirms that ultra high-performance LC (UHPLC)-tandem MS can provide reliable measurements of free and conjugated steroid concentrations in blood serum to complement those obtained by GC-MS/MS on free steroids in urine for the determination of the steroid profile required by WADA for the athlete biological passport. Furthermore, they illustrate the application of the method in the evaluation of females with mild hyperandrogenism and a group with normal circulating androgen levels, and suggest the clinical value of such an investigation as well as helping distinguish physiological outliers from sports cheats [11].

Other advances in antidoping bioanalytical approaches that could not be fitted in to this issue include, of course, the use of isotope ratio MS and advances in chromatography especially supercritical fluid chromatography of which the latter is showing great promise for routine use in doping control analysis. I hope that you, like me, will find reading the articles in this volume both interesting and rewarding.

Financial \& competing interests disclosure

The author has no relevant affiliations or financial involvement with any organization or entity with a financial interest in or financial conflict with the subject matter or materials discussed in the manuscript. This includes employment, consultancies, honoraria, stock ownership or options, expert testimony, grants or patents received or pending, or royalties.

No writing assistance was utilized in the production of this manuscript.

\section{References}

1. Aikin A, Baume N, Equey T, Rabin O. Biomarkers of doping: uses, discovery and validation. Bioanalysis 12(11), 791-800 (2020).

2. Botrè F, Georgakopoulos C, Elrayess MA. Metabolomics and doping analysis: promises and pitfalls. Bioanalysis 12(11), 719-722 (2020).

3. Moncrieffe D. Protein quantification relevant to antidoping. Bioanalysis 12(11), 711-714 (2020).

4. Fedoruk MN. Virtual drug testing: redefining sample collection in a global pandemic. Bioanalysis 12(11), 715-718 (2020).

5. López S, Meirelles J, Rayol V et al. Gene doping and genomic science in sports: where are we? Bioanalysis 12(11), 801-810 (2020).

6. Baoutina A. A brief history of the development of a gene doping test. Bioanalysis 12(11), 723-727 (2020).

7. Mongongu C, Moussa EM, Semence F et al. Use of capillary dried blood for quantification of intact IGF-I by LC-HRMS for anti-doping analysis. Bioanalysis 12(11), 737-751 (2020). 
8. Protti M, Mandrioli R, Mercolini L. Microsampling and LC-MS/MS for anti-doping testing of glucocorticoids in urine. Bioanalysis 12(11), 769-782 (2020).

9. Loria F, Manfredi M, Reverter-Branchat G, Segura J, Kuuranne T, Leuenberger N. Automation of RNA-based biomarker extraction from dried blood spots for the detection of blood doping. Bioanalysis 12(11), 729-736 (2020).

10. He G, Sheng L, Zhang J et al. Enantiomeric analysis of clenbuterol in Chinese people by LC-MS/MS to distinguish doping abuse from meat contamination. Bioanalysis 12(11), 783-790 (2020).

11. Salamin O, Ponzetto F, Cauderay M et al. Development and validation of an UHPLC-MS/MS method for extended serum steroid profiling in female populations. Bioanalysis 12(11), 753-768 (2020). 